\title{
Annual modulation of the Galactic binary confusion noise bakground and LISA data analysis
}

\author{
Naoki Seto \\ Theoretical Astrophysics, MC 130-33, California Institute of Technology, Pasadena, CA 91125
}

\begin{abstract}
We study the anisotropies of the Galactic confusion noise background and its effects on LISA data analysis. LISA has two data streams of the gravitational waves signals relevant for low frequency regime. Due to the anisotropies of the background, the matrix for their confusion noises has offdiagonal components and depends strongly on the orientation of the detector plane. We find that the sky-averaged confusion noise level $\sqrt{S(f)}$ could change by a factor of 2 in three months, and would be minimum when the orbital position of LISA is either around the spring or autumn equinox.
\end{abstract}

\section{INTRODUCTION}

The Laser Interferometer Space Antenna (LISA) is planned to be launched around 2011 and is expected to establish a new window in the low frequency gravitational wave astronomy from $0.1 \mathrm{mHz}$ to $100 \mathrm{mHz}$ [1, 2]. Its main astrophysical targets are Galactic binaries and cosmological massive black holes (MBHs) that merge with other MBHs or capture compact objects. As for the Galactic binaries, there would be a lot of sources in the LISA band. For example we will be able to resolve several thousand close white dwarf binaries at $f \gtrsim 3 \mathrm{mHz}$ [3, 4]. At lower frequency regime $f \lesssim 3 \mathrm{mHz}$ they are highly overlapped in the frequency bins, and it would be difficult to resolve them individually. As a result, they form a confusion noise background whose magnitude could be larger than the detector noise at $0.1 \mathrm{mHz} \lesssim f \lesssim 3 \mathrm{mHz}[1]$. The coalescence frequency $f_{c}$ of a MBH system is given by its redshifted total mass $M_{z}$ as $f_{c} \sim 2\left(M_{z} / 2 \times 10^{6} M_{\odot}\right)^{-1} \mathrm{mHz}$. The mass function of MBHs is highly uncertain at the lower end $\sim 10^{5} M_{\odot}[\underline{5}$, and we might have to search treasurable signals from cosmological MBHs in the Galactic confusion noise background.

The spatial distribution of the Galactic binaries would trace the Galactic structure well, and the confusion noise background would be strongly anisotropic. The background itself can be regarded as a signal that would provide some information on our Galaxy 6, 7, 8]. But we should notice that there exists a more straightforward approach to probe the Galactic structure using thousands of resolved binaries whose three dimensional positions are estimated in some error boxes [ $[$ ]. In this paper we will focus on the role of the background as a noise, and study the effects of its anisotropies on the signal analysis of LISA. We do not pay attention to the normalization of the background that has been studied by other papers using an isotropic approximation of the source distribution [3].

This paper is organized as follows. In Section 2 we study the response of interferometers to the anisotropic gravitational wave background with using the long wave approximation, and formulate the noise matrix for the Galactic confusion background. In Section 3 we numerically evaluate the bakground noise as a function of the orientation of the detector, and estimate the annual modulation of the noise level for LISA. In Section 4 we discuss the anisotropies of the signal to noise ratio (SNR) of sources at a fixed distance. Section 5 is devoted to a brief summary of this paper. In the Appendix the confusion noise matrix is analyzed in a different manner from Section 2.

\section{FORMULATION}

From data streams of LISA, we can make three meaningful modes $(A, E, T)$ such that the laser frequency noise can be reduced enough and their detector noises do not correlate (see 9] and references therein, also [10]). At the low frequency region $\left(f \ll 10 \mathrm{mHz}\right.$ : determined by the arm-length of LISA, $\left.5 \times 10^{6} \mathrm{~km}\right)$ relevant for the present analysis of the Galactic binary confusion noise, the $T$-mode has much worse sensitivity than the rest. Therefore we only discuss $A$ and $E$ modes here. These two data streams $d_{I} \quad(I=A, E)$ are made by the detector noise $n_{I}^{D}$ and the response to gravitational waves $h_{I}$

$$
d_{I}=h_{I}+n_{I}^{D} .
$$

We define the detector noise spectrum $S_{I J}^{D}$ as follows 11,12

$$
\left\langle\left(n_{I}^{D}(f)\right)^{*} n_{J}^{D}\left(f^{\prime}\right)\right\rangle=\frac{1}{2} \delta\left(f-f^{\prime}\right) S_{I J}^{D}(f) .
$$


The frequency dependence is irrelevant for our analysis, and, hereafter, we omit its explicit dependence for notational simplicity. We have the following relation for the detector noise spectrum matrix

$$
S_{A A}^{D}=S_{E E}^{D}, \quad S_{A E}^{D}=0 .
$$

The three spacecrafts of LISA form a nearly equilateral triangle, but due to the symmetric combinations of data, the two responses $h_{I}(I=A, E)$ to the low frequency gravitational waves can be essentially regarded as that of two $90^{\circ}$-interferometers rotated by $45^{\circ}$ from each other, as shown in figure 1 . We take the reference coordinate system $\left(X_{D}, Y_{D}, Z_{D}\right)$. The $Z_{D}$-axis is normal to the detector plane. When we define two units vectors $\boldsymbol{l}$ and $\boldsymbol{n}$, the response of the $A$ mode is proportional to $\boldsymbol{l} \cdot H \cdot \boldsymbol{l}-\boldsymbol{n} \cdot H \cdot \boldsymbol{n}$ and the $E$ mode to $2 \boldsymbol{n} \cdot H \cdot \boldsymbol{l}$ with some tensor $H$ made from gravitational waves. It is important to note that there is one degree of freedom to the data combinations $(A, E)$ [3]. We can make the following linear combinations $\left(d_{A}\left(\phi_{f}\right), d_{E}\left(\phi_{f}\right)\right)$ made from the original modes $\left(d_{A}, d_{E}\right)$ with a two dimensional rotation matrix $R(\phi)$ as

$$
\left(\begin{array}{l}
d_{A}\left(\phi_{f}\right) \\
d_{E}\left(\phi_{f}\right)
\end{array}\right)=R\left(2 \phi_{f}\right)\left(\begin{array}{l}
d_{A} \\
d_{E}
\end{array}\right) .
$$

These new modes $\left(A\left(\phi_{f}\right), E\left(\phi_{f}\right)\right)$ are equivalent to the responses of two $90^{\circ}$-interferometers that are obtained by rotation of the original modes $(A, E)$ with angle $\phi_{f}$ around the $Z_{D}$-axis. This reflects the spin-2 nature of a gravitational wave. We can easily confirm the following relations for the detector noise spectrum

$$
S_{A A}^{D}\left(\phi_{f}\right)=S_{E E}^{D}\left(\phi_{f}\right)=S_{A A}^{D}=S_{E E}^{D}, \quad S_{A E}^{D}\left(\phi_{f}\right)=0 .
$$

Due to the simple relation (2.4) this freedom of the effective rotation does not affect our final results as we see later. Therefore we simply use the original modes $(A, E)$ for most of our analysis.

Now we move to the binary confusion noise that is made by a large number of unresolved Galactic binaries. At the low frequency regime the response of the mode $A$ to a monochromatic binary is given by two polarization modes $(+, \times)$ of gravitational waves $[1]$

$$
h_{A}=a^{+} F_{A}^{+}+a^{\times} F_{A}^{\times} .
$$

With the quadrupole formula the amplitudes $a^{+}$and $a^{\times}$are expressed as

$$
a^{+}=K\left(1+\cos ^{2} \theta_{i}\right), \quad a^{\times}=2 K \cos \theta_{i},
$$

with the inclination angle $\theta_{i}$, and the coefficient $K$ is given in terms of the distance to the binary $r$, the chirp mass $m_{c}$, and the gravitational wave frequency $f$ as

$$
K=2 \frac{G^{5 / 3} m_{c}^{5 / 3}}{r c^{4}}(\pi f)^{2 / 3} .
$$

Two functions $F_{A}^{+}$and $F_{A}^{\times}$are determined by the sky position $(\theta, \phi)$ and the polarization angle $\psi$ of the binary in the $\left(X_{D}, Y_{D}, Z_{D}\right)$ coordinate as

$$
\begin{aligned}
& F_{A}^{+}=\frac{1}{2}\left(1+\cos ^{2} \theta\right) \cos 2 \phi \cos 2 \psi-\cos \theta \sin 2 \phi \sin 2 \psi, \\
& F_{A}^{\times}=\frac{1}{2}\left(1+\cos ^{2} \theta\right) \cos 2 \phi \sin 2 \psi+\cos \theta \sin 2 \phi \cos 2 \psi .
\end{aligned}
$$

The response $h_{E}$ to the $E$ mode is given by a similar expression as eq. (2.6). We replace the azimuthal angle $\phi$ to $\phi-\pi / 4$ in eqs.(2.9) and (2.10). As in eq.(2.2) defined for the detector noise, we denote the Galactic binary noise spectrum matrix $S_{I J}^{B}$ by the responses $\left(h_{A}^{B}, h_{E}^{B}\right)$ of the modes $(A, E)$ to all the unresolved Galactic binaries. With using eq.(2.6) we sum up all the unresolved binary within Galaxy. For simplicity we assume that the binary mass function does not depend on the frequency $f$ or the position in the Galaxy. This would be a fairly reasonable assumption. Considering the nature of the confusion noise we average over the inclination and polarization angles $\theta_{i}, \psi$, the spatial position $\boldsymbol{r}$, and obtain the following expression

$$
S_{I J}^{B}=P \int d \boldsymbol{r} \rho(\boldsymbol{r}) r^{-2} F_{I J}(\theta, \phi),
$$


where $P$ is a normalization factor that is not important for our analysis. The function $\rho(\boldsymbol{r})$ is the density profile of the Galactic binaries, and the functions $F_{I J}$ are defined as

$$
\begin{aligned}
& F_{A A}=\frac{1}{2}\left[\left(\frac{1+\cos ^{2} \theta}{2} \cos 2 \phi\right)^{2}+(\cos \theta \sin 2 \phi)^{2}\right], \\
& F_{E E}=\frac{1}{2}\left[\left(\frac{1+\cos ^{2} \theta}{2} \sin 2 \phi\right)^{2}+(\cos \theta \cos 2 \phi)^{2}\right], \\
& F_{A E}=F_{E A}=\frac{1}{2}\left[\left(\frac{1+\cos ^{2} \theta}{2}\right)^{2}-\cos ^{2} \theta\right] \cos 2 \phi \sin 2 \phi .
\end{aligned}
$$

Using a spherical coordinate we can express eq.(2.11) as [6]

$$
S_{I J}^{B}=\int d \Omega B(\theta, \phi) F_{I J}(\theta, \phi),
$$

where the anisotropy of the gravitational wave background $B(\theta, \phi)$ is defined as

$$
B(\theta, \phi) \equiv P \int d r \rho(r, \theta, \phi) .
$$

If the source distribution $\rho(\boldsymbol{x})$ for gravitational waves is same as that of the light and the interstellar absorption is negligible, the function $B(\theta, \phi)$ is also the luminosity distribution of the Milky Way on the sky.

In reality there would be some fluctuations around the above expression 2.11 due to the finite number of the binaries. But this effect is beyond the scope of this paper. Some binaries would be close enough to have significant SNR above the confusion noise level, and could be removed from the background. But a rough estimation indicates that the typical distance to such binaries is smaller than $\sim 100$ pc at the low frequency region $f<1 \mathrm{mHz}$, and their subtraction would change our results only slightly [14].

For an isotropic binary distribution we can replace the function $F_{I J}$ by

$$
F_{A A}=F_{E E}=\frac{1}{5}, \quad F_{A E}=0,
$$

and the noise matrix $S_{I J}^{B}$ is diagonal (proportional to the unit matrix). For reference we define the noise amplitude $S_{i s o}^{B}$ that is obtained with using $F_{I J}=1 / 5$ in eq.(2.11). This monopole spectrum $S_{i s o}^{B}$ is what has been used in most of previous studies. Differences between $S_{I J}^{B}$ and $S_{i s o}^{B}$ due to the proper angular dependence of $F_{I J}$ are the main issue of this paper.

We can calculate the noise matrix $S_{I J}^{B}\left(\phi_{f}\right)$ for the new data combination $\left(A\left(\phi_{f}\right), E\left(\phi_{f}\right)\right)$ generated by the simple relation (2.4). It is given by the original one $S_{I J}^{B}$ as

$$
S_{I J}^{B}\left(\phi_{f}\right)=R\left(2 \phi_{f}\right) S_{I J}^{B} R\left(-2 \phi_{f}\right)
$$

Let us consider an analysis for some strong gravitational wave signal $\left(h_{A, X}, h_{E, X}\right)$ from a source $X$, e.g. the ring down waveform from a merged $\mathrm{MBH}$ binary. When the detector noise $S_{I J}^{D}$ is much smaller than the Galactic confusion noise $S_{I J}^{B}$, the SNR is given as

$$
S N R^{2} \propto\left(h_{A}, h_{E}\right)^{*}\left(S^{B}\right)^{-1}\left(\begin{array}{c}
h_{A} \\
h_{E}
\end{array}\right) .
$$

From eqs. 2.4 and (2.19) we can easily confirm that the freedom of the rotation angle $\phi_{f}$ is completely killed in the above relation for SNR. This is the reason we can forget it from the beginning. But it is instructive to take a rotation angle $\phi_{f}=\phi_{0}$ so that the noise matrix $S_{I J}^{B}\left(\phi_{0}\right)$ is diagonalized as

$$
\left(\begin{array}{cc}
\lambda_{1} & 0 \\
0 & \lambda_{2}
\end{array}\right)
$$

Then we have the following simple relation

$$
S N R^{2} \propto \frac{\left|h_{A}\left(\phi_{0}\right)\right|^{2}}{\lambda_{1}}+\frac{\left|h_{E}\left(\phi_{0}\right)\right|^{2}}{\lambda_{2}} .
$$


Next we evaluate the effective noise level for the two modes by averaging out the direction and orientation the source $X$. Apparently the averages for $\left|h_{A X}\left(\phi_{f}\right)\right|^{2}$ and $\left|h_{E X}\left(\phi_{f}\right)\right|^{2}$ do not depend on the choice of the angle $\phi_{f}$, and we have $\left\langle\left|h_{A X}\left(\phi_{0}\right)\right|^{2}\right\rangle=\left\langle\left|h_{E X}\left(\phi_{0}\right)\right|^{2}\right\rangle$. Thus it is reasonable to define the effective noise level $S_{\text {eff }}^{B}$ by the following equation

$$
S_{e f f}^{B}=2\left(\frac{1}{\lambda_{1}}+\frac{1}{\lambda_{2}}\right)^{-1}=2 \frac{\operatorname{det} S^{B}}{\operatorname{tr} S^{B}} .
$$

The coefficient 2 comes from the number of the modes. The inverse of this quantity is proportional to the averaged $S N R^{2}$ for sources at a fixed distance. For ground-base detectors, such as, LIGO II, the effective distance $d_{e}$ to a source that can be detected above a given SNR threshold is often used to characterize the noise level. When the cosmological effects for the event rate is small $\left(e . g . d_{e} \sim 300 \mathrm{Mpc}\right)$, this is a intuitive measure for a detector. But for the cosmological sources the situation is not so simple. Therefore we do not pursuit such a kind of measure, but use the effective noise $S_{e f f}^{B}$. This approach greatly simplifies the treatment of the polarization angle.

Finally we make a brief mathematical comment on the effective noise level $S_{e f f}^{B}$. A rigid analysis is given in the Appendix. The functions $F_{I J}(\theta, \phi)$ are written by spherical harmonic functions $Y_{l m}(\theta, \phi)$ with $l=0,2$ and 4 [7, [8]. This means that the angular dependence of the Galactic confusion noise felt by the detectors $(A, E)$ is limited to these three modes at low frequency region. Therefore the effective noise for arbitrary detector plane is determined by $1+5+9=15$ real parameters that characterize anisotropies of the binary noise (total of $5+9$ ) and the monopole mode (total of 1). The monopole mode is nothing but the isotropic component $S_{i s o}^{B}$.

\section{ANNUAL MODULATION OF THE EFFECTIVE NOISE}

Due to the annual rotation of the detector plane of LISA, the effective noise $S_{\text {eff }}^{B}$ changes with time. To denote the orbital phase of LISA we use the time $T$ measured from the autumn equinox point in units of year. The actual date for $T=0$ corresponds to $\sim 20$ days after the autumn equinox day, as LISA trails $20^{\circ}$ behind the Earth. With eq. (2.11) we can calculate the annual modulation of the noise $S_{\text {eff }}^{B}$ at given moment $T$. The detector plane inclines to the ecliptic plane by $60^{\circ}$. We assume that the vertex of the cone made by the envelope of the detector planes exists at south as shown in figure 2. Hereafter we implicitly assume this configuration. When we take it to the opposite direction, the time dependence of the noise $S_{\text {eff }}^{B}$ changes by $T=0.5 y$ from our results. In figure 2 we also define the ecliptic coordinate that is very useful for describing the motion of LISA. In this coordinate the directions of the Galactic center, the north and south Galactic poles are $(\theta, \phi)=\left(-95.5^{\circ},-93.2^{\circ}\right),\left(60.4^{\circ}, 180.0^{\circ}\right)$ and $\left(119.6^{\circ}, 0.0^{\circ}\right)$ [15].

\section{A. Galactic model}

First we calculate the all sky map of the gravitational wave luminosity $B(\theta, \phi)$. We mainly use the Galactic stellar distribution model given in [16]. This model (hereafter BGS model) contains both the triaxial bulge and disk components obtained by fitting the near infrared COBE/DIRBE surface brightness map. In addition to these two components we have also studied contribution of the halo stars. But its effect is almost negligible for reasonable model parameters, and we do not discuss the halo component in this paper (see also [6]). The explicit form of the BGS model is given as

$$
\rho(\boldsymbol{x})=C\left(f_{b}(\boldsymbol{x})+f_{d}(\boldsymbol{x})\right),
$$

where $C$ is a normalization constant and

$$
\begin{aligned}
f_{b} & =f_{0} \frac{e^{-a^{2} / a_{m}^{2}}}{\left(1+a / a_{0}\right)^{1.8}} \\
f_{d} & =\left(\frac{e^{-|z| / z_{0}}}{z_{0}}+\alpha \frac{e^{-\mid z_{\mid} / z_{1}}}{z_{1}}\right) R_{s} e^{-R / R_{s}}, \\
a & \equiv\left(x^{2}+\frac{y^{2}}{\eta^{2}}+\frac{z^{2}}{\zeta^{2}}\right) \\
R & \equiv\left(x^{2}+y^{2}\right)^{1 / 2}
\end{aligned}
$$

Here we use a Galactic coordinate. Its $z$-axis is oriented to the north Galactic pole, and the direction of the $x$-axis (the long axis of the triaxial bulge) is $20^{\circ}$ different from the Sun-center line. In this model the solar system exists at 
$R=8.0 \mathrm{kpc}$ and $z=14 \mathrm{pc}$. The parameters are given as $f_{0}=624, a_{m}=1.9 \mathrm{kpc}, a_{0}=100 \mathrm{pc}, R_{s}=2.5 \mathrm{kpc}, z_{0}=210 \mathrm{pc}$, $z_{1}=42 \mathrm{pc}, \alpha=0.27, \eta=0.5$ and $\zeta=0.6$. We performed numerical integration of eq. (2.16) and obtain the map $B(\theta, \phi)$ as shown in figure 3 where the ecliptic coordinate is used. We can observe the bulge structure around the Galactic center. Note that, for the configuration of figure 2 , the $Z_{D}$-axis of LISA passes pretty close to the north Galactic pole around the autumn equinox.

\section{B. noise map}

With the map $B(\theta, \phi)$ of the gravitational wave anisotropies the effective noise map $S_{I J}^{B}$ is obtained by convolution of the response functions $F_{I J}$. In figure 3 the noise map $S_{e f f}^{B}$ is given as a function of the orientation of the $Z_{D}$-axis (with angles $(\theta, \phi)$ in the ecliptic coordinate) of the detector. Two orientations $(\theta, \phi)$ and $(\pi-\theta, \phi+\pi)$ give a same value due to the symmetry of the response functions $F_{I J}$. The $Z_{D}$-axis of LISA moves along the dot-dashed line. In the map the maximum value of $S_{e f f}^{B} / S_{i s o}^{B}$ is 1.69 around the direction of the Galactic center, and minimum value is 0.38 around the Galactic poles. As mentioned before the $Z_{D}$-axis of LISA passes pretty close to the poles at the autumn equinox. This means that the effective noise at the autumn equinox is almost the smallest value in the map. We can also see that the effective noise is higher in summer than winter. The separation between the $Z_{D}$-axis and the Galactic center on the sky is smaller for the former. The map traces the stellar distribution along the Galactic disk (Milky Way on the sky), but is not as significant as figure 3 .

For the BGS model we also analyzed the deformation of the noise matrix $S_{I J}^{B}$ from a following simple shape that is proportional to the unit matrix

$$
\left(\begin{array}{ll}
\lambda & 0 \\
0 & \lambda
\end{array}\right)
$$

This deviation is caused by the hexadecupole $(l=4)$ mode of the background $B(\theta, \phi)$ (see Appendix). We numerically

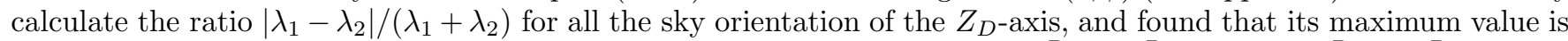
0.152. Therefore it is not a bad approximation to put $\lambda_{1}=\lambda_{2}=\lambda$, namely $S_{A A}^{B}=S_{E E}^{B}=\lambda$ and $S_{A E}^{B}=S_{E A}^{B}=0$. In this approximation the noise matrix is characterized by a single quantity $\lambda(\theta, \phi)$ and Figure 3 can be roughly regarded as its map.

We have adopted the BGS model for the spatial distribution of the Galactic binaries. But the radial scale length of the disk $R_{s}$ has some uncertainties [17]. Furthermore the distribution of the gravitational wave sources might be different from that of the near infrared light. Here we study how the effective noise changes with the model parameter. We use the following exponential disk model for the Galactic density profile $\rho(\boldsymbol{r})$ [17]

$$
\rho(R, z)=n_{0} \exp \left[-\left(R / R_{s}\right)\right] \operatorname{sech}^{2}\left[\left(z / z_{s}\right)\right],
$$

with the Galactic cylindrical coordinate $(R, z)$ and a normalization constant $n_{0}$. The radial scale length $R_{s}$ has typically $R_{s}=1.7 \sim 3 \mathrm{kpc}$. In this model we fix the disk scale height at $z_{s}=200 \mathrm{pc}$, and put the solar system at $R=8.5 \mathrm{kpc}$ and $z=30 \mathrm{pc}$.

In figure 4 we show our numerical results for the normalized noise level $S_{\text {eff }}^{B} / S_{\text {iso }}^{B}$ as a function of the time $T$. We choose the scale length $R_{s}$ at $R_{s}=1.0,1.7$ and $2.5 \mathrm{kpc}$. The results for $R_{s}=2.5 \mathrm{kpc}$ and the BGS model are almost the same. As in figure 2 the Galactic center is nearly on the ecliptic plane and also nearly normal to the two equinox directions. Let us first consider a simple situation that (i) the density profile is a delta function $\delta^{3}\left(\boldsymbol{r}-\boldsymbol{r}_{G C}\right)$ around the Galactic center $\boldsymbol{r}_{G C}$, and (ii) the center is on the detector plane with angles $(\theta, \phi)=\left(\pi / 2, \phi_{G C}\right)$ in the $\left(X_{D}, Y_{D}, Z_{D}\right)$ coordinate system in figure 1 . Then the mode $A\left(\phi_{G C}\right)$ is completely free from the Galactic binary noise and one of the eigen values $\lambda_{1}$ vanishes. Thus the effective noise becomes $S_{\text {eff }}^{B}=0$ from eq.(2.22). In reality the density profile $\rho(\boldsymbol{r})$ has a three dimensional structure and the eigen values $\lambda_{i}$ take finite values. But the density profile are highly concentrated around the center $\boldsymbol{r}_{G C}$, and the minimum values of $S_{\text {eff }}^{B} / S_{\text {iso }}^{B}$ becomes smaller as we decrease the scale length $R_{s}$ as shown in figure 5. The deformation $\left|\lambda_{1}-\lambda_{2}\right| /\left(\lambda_{1}+\lambda_{2}\right)$ is larger for a smaller length $R_{s}$.

As the effective noise level $S_{e f f}^{B}$ changes with time $T$, it would be useful to define a time average of the noise. The most natural definition from the standpoint of the signal analysis would be the following one

$$
\bar{S}_{e f f}^{B} \equiv\left(\frac{1}{1 \mathrm{yr}} \int_{0}^{1} \frac{d T}{S_{e f f}^{B}(T)}\right)^{-1} .
$$

This quantity is inversely proportional to the all sky average of $S N R^{2}$ for sources at a fixed distance as the effective noise $S_{e f f}^{B}$. We have $\bar{S}_{e f f}^{B} / S_{i s o}^{B}=1.54$ and 1.15 for $R_{s}=1.0$ and $2.5 \mathrm{kpc}$ respectively. Therefore the traditional estimation based on the monopole mode gives a fairly good result for the time averaged noise. 


\section{SNR AS A FUNCTION OF THE SKY POSITION}

So far we have discussed how the binary noise changes with the orientation of the detector. In this section we first analyze the dependence of SNR on the sky position of a monochromatic source with a fixed distance and 1yr integration time. We denote the source direction $\left(\theta_{s}, \phi_{s}\right)$ in the ecliptic coordinate. With the rotation of LISA, the noise matrix $S_{I J}^{B}(t)$ and the response function $F_{A, E}^{+, \times}$both change with time. At a given moment, the direction of the binary $\left(\theta_{D}, \phi_{D}\right)$ in the rotating detector coordinate $\left(X_{D}, Y_{D}, Z_{D}\right)$ can be formally expressed as $\left(\theta_{D}\left(\theta_{s}, \phi_{s}, t\right)\right.$, $\left.\phi_{D}\left(\theta_{s}, \phi_{s}, t\right)\right)$. Then the quantity $S N R^{2}$ with averaging over the source orientation (polarization and inclination) is proportional to the following time integral

$$
G\left(\theta_{s}, \phi_{s}\right)=\int_{1 \mathrm{yr}} \sum_{I J} F_{I J}\left(\theta_{D}\left(\theta_{s}, \phi_{s}, t\right), \phi_{D}\left(\theta_{s}, \phi_{s}, t\right)\right)\left(S_{I J}^{B}(t)\right)^{-1} d t
$$

Actually the polarization angle $\psi$ in eqs.(2.9) and (2.10) also changes with time. But its average commutes with the time integration.

To deal with the complicated time dependence of the response function $F_{I J}$ and the noise matrix $S_{I J}^{B}(t)$ we use the following three prescriptions with

(1) the monopole noise model $S_{I J}^{B}(t)=S_{i s o}^{B} \delta_{I J}$ and the angular averaged response functions $F_{I J}=\delta_{I J} / 5$,

(2) the monopole noise model $S_{I J}^{B}(t)=S_{i s o}^{B} \delta_{I J}$ but including the time dependence of the response functions $F_{I J}$,

(3) including the time dependence for both the noise matrix $S_{I J}^{B}(t)$ and the response functions $F_{I J}$.

We denote the integral $G$ with above prescriptions as $G_{1}, G_{2}$ and $G_{3}$ respectively. The method (2) has been sometimes used for study of the signal analysis that would be valid at higher frequency regime where the detector noise dominant [18]. As the configuration of LISA is symmetric around the $Z_{E}$-axis of the ecliptic coordinate (figure 2 ) and the freedom of the $\phi_{f}$-rotation is irrelevant here, the result $G_{2}\left(\theta_{s}, \phi_{s}\right)$ should depend only on the zenith angle $\theta_{s}$. We can derive a simple analytic expression for the ratio $G_{2} / G_{1}$ as follows

$$
\frac{G_{2}\left(\theta_{s}\right)}{G_{1}}=\frac{15605-2300 \cos 2 \theta_{s}-185 \cos 4 \theta_{s}}{16384} .
$$

The function $G_{2}\left(\theta_{s}\right) / G_{1}$ becomes maximum 1.08 at $\theta_{s}=\pi / 2$ and minimum 0.80 at $\theta_{s}=0$ and $\pi$. Due to the angular average of the response function by the annual rotation of LISA, the simple prescription (1) gives fairly good results for the isotropic noise. We can easily confirm that the angular average of the ratio $G_{2}\left(\theta_{s}\right) / G_{1}$ becomes unity.

Next we calculate the ratio $G_{3}\left(\theta_{s}, \phi_{s}\right) / G_{1}$ by numerical integration of the expression (4.1). The results for all sky direction are shown in figure 6. We used the same Galactic model as figure 3. The ratio becomes maximum value 1.56 around the Galactic poles and the minimum 0.86 (not at the direction of the Galactic center). The ratio $G_{3} / G_{1}$ is a convolution of the sky dependence of the response function $F_{I J}$ and the time varying noise $S_{I J}^{B}(t)$ whose profile is shown in figures 4 and 5 . To interpret figure 6 , let us regard the expression (4.1) as a integral of the function $S_{I J}^{B}(t)^{-1}$ with the weight $F_{I J}$. The function $S_{I J}^{B}(t)^{-1}$ becomes maximum when the $Z_{D^{-}}$axis of the detector is nearly oriented to the Galactic poles as shown in figures 3 and 4 . At this epoch the weight function $F_{I J}$ is largest to the direction of the Galactic poles and and smallest to the Galactic disk, if we assume the matrix $S_{I J}^{B}(t)^{-1}$ in the form $(3.6)$.In this manner the map traces the Galactic structure well. We should also notice that the overall $\theta_{s}$ dependence of the ratio $G_{3} / G_{1}$ is similar to the ratio $G_{2} / G_{1}$, as easily expected.

We can make a similar argument for a short-lived signal such as the ring down wave from a MBH binary. As mentioned above, the best time is the autumn equinox and the best source directions at that time are the Galactic poles (both $F_{I J}$ and $S_{I J}^{B}(t)^{-1}$ are maximum). The worst season of the effective noise is summer when the orientation of the $Z_{D}$-axis is nearly on the Galactic disk. At this configuration the response function $F_{I J}$ takes the smallest value

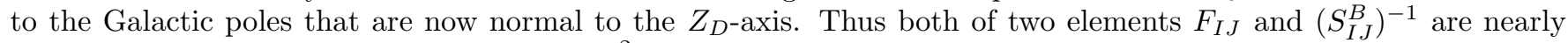
minimized. As a results the SNR (not $S N R^{2}$ ) at the direction of Galactic poles changes by a factor of 4 in three month. A factor of 2 comes from the changes of the noise and another factor of 2 comes from that of the response function. Both of two effects go to the same direction to amplify the time modulation.

\section{SUMMARY}

In this paper we have studied the anisotropies of the Galactic confusion noise background and its effect on the data analysis of LISA at the low frequency regime $f \lesssim 3 \mathrm{mHz}$. In contrast to the traditional monopole approximation the anisotropies induce the correlation of confusion noises between the $A$ and $E$ modes of LISA, and the effective noise level depends strongly on the time. In the followings we briefly summarize our results. 
The effective noise level of LISA becomes smallest around the autumn equinox, and largest in summer for the configuration in figure 2. The difference of the noise $\sqrt{S_{\text {eff }}^{B}}$ at these two epochs is a factor of 2 . The detector plane of LISA at the autumn equinox is almost normal to the Galactic poles and the effective noise level is very close to the global minimum of the noise map given for the all possible orientations of the detector. The time average of the effective noise is different from the traditional monopole approximation by less than $50 \%$.

We have also analyzed the dependence of the SNR on the sky position of sources with a fixed distance and averaged orientation. For a long lived source the dependence after 1yr integration is weak, and fluctuations on the sky are $\sim 30 \%$ level. This is mainly due to the angular average of the response function by annual rotation of LISA. For a short lived source the SNR at a given direction changes strongly with time. For example the SNR of a source at the direction of the Galactic pole changes by a factor of $\sim 4$ in three months. A factor of 2 comes from the modulation of the confusion noise level and another factor of 2 is from that of the angular response function of the detector.

\section{Acknowledgments}

The author thanks Asantha Cooray and Sterl Phinney for valuable discussions. This work is supported in part by NASA grant NAG5-10707.

\section{APPENDIX A: SPHERICAL HARMONIC EXPANSION}

The noise matrix $S_{I J}^{B}$ is determined by the orientation of the coordinate system $K_{D} ;\left(Z_{D}, Y_{D}, Z_{D}\right)$ (as defined in figure 1) that would change with time. In this situation the spherical harmonic expansion for a fixed coordinate system $K_{0} ;\left(X_{0}, Y_{0}, Z_{0}\right)$ (e.g. the ecliptic coordinate in figure 2) would be useful [7]. We can related two systems $K_{D}$ and $K_{0}$ by the Euler angles $(\alpha, \beta, \gamma)$. Here $(\alpha, \beta)$ is the direction of the $Z_{D}$-axis in the $K_{0}$ system, and $\gamma$ is essentially corresponds to the freedom of the $\phi_{f}$-rotation in eq.(2.4) around the $Z_{D}$-axis. We denote the noise matrix in the $K_{D}$ system as $S_{I J}^{B}(\alpha, \beta, \gamma)$. In the configuration of figure 2 we can parameterize the motion of LISA with $\alpha=2 \pi T$, $\beta=-\pi / 3$ and $\gamma=-2 \pi T+\gamma_{0}$ with some constant $\gamma_{0}$ and the orbital time $T$.

The angular dependence of the gravitational wave intensity (eq.(2.16) $) B\left(\theta_{0}, \phi_{0}\right)$ is decomposed by the spherical harmonics $Y_{l m}\left(\theta_{0}, \phi_{0}\right)$ as

$$
B\left(\theta_{0}, \phi_{0}\right)=\sum_{l, m} B_{l m} Y_{l m}\left(\theta_{0}, \phi_{0}\right)
$$

Here the coefficients $B_{l m}$ is given as

$$
B_{l m}=\langle l m \mid B\rangle,
$$

where we have used the traditional notation for the inner product $\langle a \mid b\rangle \equiv \int d \Omega a^{*}\left(\theta_{0}, \phi_{0}\right) b\left(\theta_{0}, \phi_{0}\right)$, and an abbreviation $|l m>\equiv| Y_{l m}\left(\theta_{0}, \phi_{0}\right)>$. Our goal is to write down the matrix $S_{I J}^{B}(\alpha, \beta, \gamma)$ with using the coefficients $B_{l m}$.

First the matrix $S_{I J}^{B}(\alpha, \beta, \gamma)$ is formally given as follows

$$
S_{I J}^{B}(\alpha, \beta, \gamma)=\left\langle F_{I J}\left(\theta_{0}, \phi_{0}\right)\left|U(\alpha, \beta, \gamma)^{-1}\right| B\left(\theta_{0}, \phi_{0}\right)\right\rangle,
$$

where $U(\alpha, \beta, \gamma)$ is the rotation operator with the Eular angles $\alpha, \beta$ and $\gamma$. Using the identity $\sum_{l m}|\operatorname{lm}><\operatorname{lm}|=1$ for the complete sets $\mid l m>$, we have

$$
\begin{aligned}
S_{I J}^{B}(\alpha, \beta, \gamma) & =\sum_{l m} \sum_{l^{\prime} m^{\prime}}\left\langle F_{I J}\left(\theta_{0}, \phi_{0}\right) \mid l m\right\rangle\left\langle l m\left|U(\alpha, \beta, \gamma)^{-1}\right| l^{\prime} m^{\prime}\right\rangle\left\langle l^{\prime} m^{\prime} \mid B\left(\theta_{0}, \phi_{0}\right)\right\rangle, \\
& =\sum_{l m m^{\prime}}\left\langle F_{I J}\left(\theta_{0}, \phi_{0}\right) \mid l m\right\rangle\left\langle\operatorname{lm}\left|U(\alpha, \beta, \gamma)^{-1}\right| l m^{\prime}\right\rangle\left\langle l m^{\prime} \mid B\left(\theta_{0}, \phi_{0}\right)\right\rangle .
\end{aligned}
$$

Next we calculate the coefficients $C_{I J l m} \equiv\left\langle F_{I J} \mid l m\right\rangle$ for the angular dependence of the response functions. We have $C_{I J l m}=0$ for $l \neq 0,2$ and 4 . The followings are all of the non-vanishing coefficients;

$$
\begin{aligned}
& C_{A A 00}=C_{E E 00}=\frac{2 \sqrt{\pi}}{5} \\
& C_{A A 20}=C_{E E 20}=\frac{4}{7} \sqrt{\frac{\pi}{5}}
\end{aligned}
$$




$$
\begin{aligned}
& C_{A A 40}=C_{E E 40}=\frac{\sqrt{\pi}}{105} \\
& C_{A A 44}=C_{A A 4-4}=-C_{E E 44}=-C_{E E 4-4}=\frac{1}{3} \sqrt{\frac{\pi}{70}} \\
& C_{A E 44}=-C_{A E 4-4}=\frac{i}{3} \sqrt{\frac{\pi}{70}} .
\end{aligned}
$$

Then eq. A5 is simplified to

$$
\begin{aligned}
S_{I J}^{B}(\alpha, \beta, \gamma)= & B_{00}\left\langle 00\left|U(\alpha, \beta, \gamma)^{-1}\right| 00\right\rangle C_{I J 00}+\sum_{m} B_{2 m}\left\langle 20\left|U(\alpha, \beta, \gamma)^{-1}\right| 2 m\right\rangle C_{I J 20} \\
& +\sum_{m} B_{4 m}\left\langle 40\left|U(\alpha, \beta, \gamma)^{-1}\right| 4 m\right\rangle C_{I J 40}+\sum_{m} B_{4 m}\left\langle 44\left|U(\alpha, \beta, \gamma)^{-1}\right| 4 m\right\rangle C_{I J 44} \\
& +\sum_{m} B_{4 m}\left\langle 4-4\left|U(\alpha, \beta, \gamma)^{-1}\right| 4 m\right\rangle C_{I J 4-4}
\end{aligned}
$$

For further calculation we use the relation between the matrix elements $\left\langle\operatorname{lm}\left|U(\alpha, \beta, \gamma)^{-1}\right| m^{\prime}\right\rangle$ and the spin-weight spherical harmonics ${ }_{s} Y_{l m}(\alpha, \beta)$ as

$$
\left\langle l m\left|U(\alpha, \beta, \gamma)^{-1}\right| l m^{\prime}\right\rangle=e_{-m}^{i \gamma m} Y_{l m^{\prime}}(\beta, \alpha) \sqrt{\frac{4 \pi}{2 l+1}} .
$$

After some calculation we finally obtain the explicit forms for the matrix $S_{I J}^{B}(\alpha, \beta, \gamma)$ as

$$
\begin{aligned}
S_{A A}^{B} & =\frac{2 \sqrt{\pi}}{5} B_{00}+\frac{8 \pi}{35} B_{2 m} Y_{2 m}(\beta, \alpha)+\frac{2 \pi}{315} B_{4 m} Y_{4 m}(\beta, \alpha)+\frac{2 \pi}{9 \sqrt{70}} B_{4 m}\left[{ }_{4} Y_{4 m}(\beta, \alpha) e^{4 i \gamma}+{ }_{4} Y_{4 m}(\beta, \alpha) e^{-4 i \gamma}\right] \mathrm{A} 1{ }^{4} \\
S_{E E}^{B} & =\frac{2 \sqrt{\pi}}{5} B_{00}+\frac{8 \pi}{35} B_{2 m} Y_{2 m}(\beta, \alpha)+\frac{2 \pi}{315} B_{4 m} Y_{4 m}(\beta, \alpha)-\frac{2 \pi}{9 \sqrt{70}} B_{4 m}\left[{ }_{4} Y_{4 m}(\beta, \alpha) e^{4 i \gamma}+{ }_{4} Y_{4 m}(\beta, \alpha) e^{-4 i \gamma}\right]\left(\mathrm{A}_{1} 1\right. \\
S_{A E}^{B} & =\frac{2 \pi i}{9 \sqrt{70}} B_{4 m}\left[{ }_{4} Y_{4 m}(\beta, \alpha) e^{4 i \gamma}-{ }_{4} Y_{4 m}(\beta, \alpha) e^{-4 i \gamma}\right]
\end{aligned}
$$

where the summation with respect to the index $m$ is implicitly assumed. These symmetric expressions would be quite useful to study how we can estimate the anisotropies $B_{l m}$ form the data $S_{I J}^{B}$. The correlation analysis $S_{A E}^{B}$ can be used to extract the hexadecupole $(l=4)$ mode and the freedom of adjusting $\gamma$ would be important here. Detailed studies on this issue would be presented elsewhere.

We can easily confirm that both $\operatorname{tr} S_{I J}^{B}$ and $\operatorname{det} S_{I J}^{B}$ do not depend on the angle $\gamma$, as expected. We can derive some expressions for the eigen values $\lambda_{1}$ and $\lambda_{2}$ of the noise matrix $S_{I J}^{B}$. With notation $(a(\beta, \alpha))_{a v}$ for angular average of a function $a(\beta, \alpha)$ over a unit sphere we have

$$
\begin{aligned}
\frac{1}{2}\left(\lambda_{1}+\lambda_{2}\right)_{a v} & =\frac{2 \sqrt{\pi}}{5} B_{00} \\
\frac{1}{4}\left(\left(\lambda_{1}+\lambda_{2}\right)^{2}\right)_{a v} & =\frac{4 \pi}{25} B_{00}^{2}+\frac{16 \pi}{35^{2}} \sum_{m}\left|B_{2 m}\right|^{2}+\frac{\pi}{315^{2}} \sum_{m}\left|B_{4 m}\right|^{2}, \\
\frac{1}{4}\left(\left(\lambda_{1}-\lambda_{2}\right)^{2}\right)_{a v} & =\frac{2 \pi}{2835} \sum_{m}\left|B_{4 m}\right|^{2} .
\end{aligned}
$$

[1] P. L. Bender et al., LISA Pre-Phase A Report, Second edition, July 1998.

[2] C. Cutler and K. S. Thorne, arXiv:gr-qc/0204090

[3] D Hils, P. L. Bender and R. F. Webbink Astrophys. J. 360, 75 (1990); G. Nelemans, L. R. Yungelson and S. F. Portegies Zwart, arXiv:astro-ph/0105221

[4] P. L. Bender and D. Hils, Class. Quant. Grav. 14, 1439 (1997); N. J. Cornish and S. L. Larson, Phys. Rev. D 67, 103001 (2003)

[5] L. Ferrarese and D. Merritt, Astrophys. J. 539, L9 (2000); K. Gebhardt et al., Astrophys. J. 539, L13 (2000).

[6] G. Giampieri and A. G. Polnarev Mon. Not. R. Astron. Soc. 291, 149 (1997); G. Giampieri and A. G. Polnarev, Class. Quant. Grav. 14, 1521 (1997). 


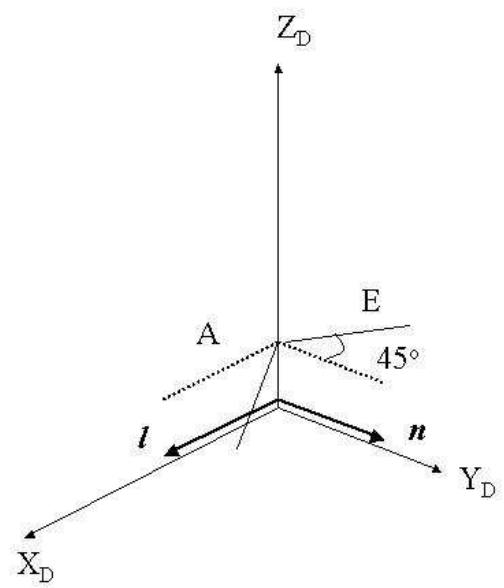

FIG. 1: Definition of the detector coordinate $\left(X_{D}, Y_{D}, Z_{D}\right)$. The $Z_{D}$-axis is normal to the detector plane. A and E modes can be regarded as two L-shaped detectors rotated by $45^{\circ}$.

[7] N. J. Cornish, Class. Quant. Grav. 18, 4277 (2001)

[8] C. Ungarelli and A. Vecchio, Phys. Rev. D 64, 121501 (2001)

[9] T. A. Prince, M. Tinto,S. L. Larson and J. W. Armstrong, Phys. Rev. D 66, 122002 (2002)

[10] N. J. Cornish and R. W. Hellings, Class. Quant. Grav. 20, 4851 (2003) D. A. Shaddock, M. Tinto, F. B. Estabrook and J. W. Armstrong, Phys. Rev. D 68, 061303 (2003)

[11] K. S. Thorne, in 300 Years of Gravitation, edited by S. W. Hawking and W. Israel (Cambridge, England, 1987), pp.330-458.

[12] C. Cutler and E. E. Flanagan, Phys. Rev. D 49, 2658 (1994)

[13] K. R. Nayak, S. V. Dhurandhar, A. Pai and J. Y. Vinet, Phys. Rev. D 68, 122001 (2003)

[14] N. Seto, Mon. Not. Roy. Astron. Soc. 333, 469 (2002)

[15] J. Binney and M. Merrifield, Galactic Astronomy (Princeton University Press, Princeton, 1998).

[16] J. Binney, O. Gerhard and D. Spergel, Mon. Not. Roy. Astron. Soc. 288, 365 (1997)

[17] P. D. Sackett, Astrophys. J. 483, 103 (1997)

[18] C. Cutler, Phys. Rev. D 57, 7089 (1998).

[19] J. N. Goldberg, A. J. MacFarlane, E. T. Newman, F. Rohrlich and E. C. G. Sudarshan, J. Math. Phys. 8, 2155 (1967); W. Hu and M. White, Phys. Rev. D 56, 596 (1997) 

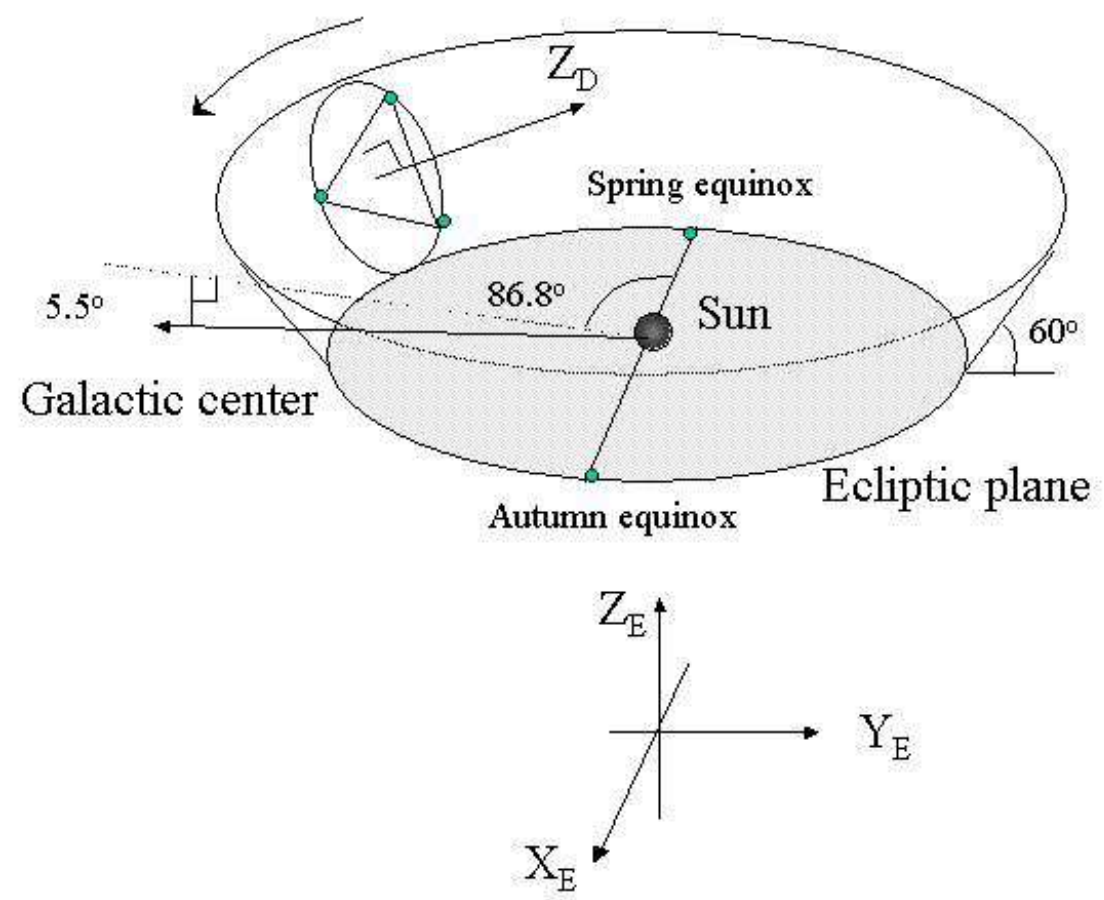

FIG. 2: Configuration of LISA and the definition of the ecliptic coordinate $\left(X_{E}, Y_{E}, Z_{E}\right)$. The $Z_{E}$-axis is normal to the ecliptic plane and $X_{E}$-axis is oriented to the autumn equinox. The direction of the Galactic center is $\theta=95.5^{\circ}$ and $\phi=-93.2^{\circ}$ in this coordinate. With this configuration the azimuthal angle for the orientation of the $Z_{D}$-axis and that for the direction of the detector (from the Sun) differ by $180^{\circ}$. 


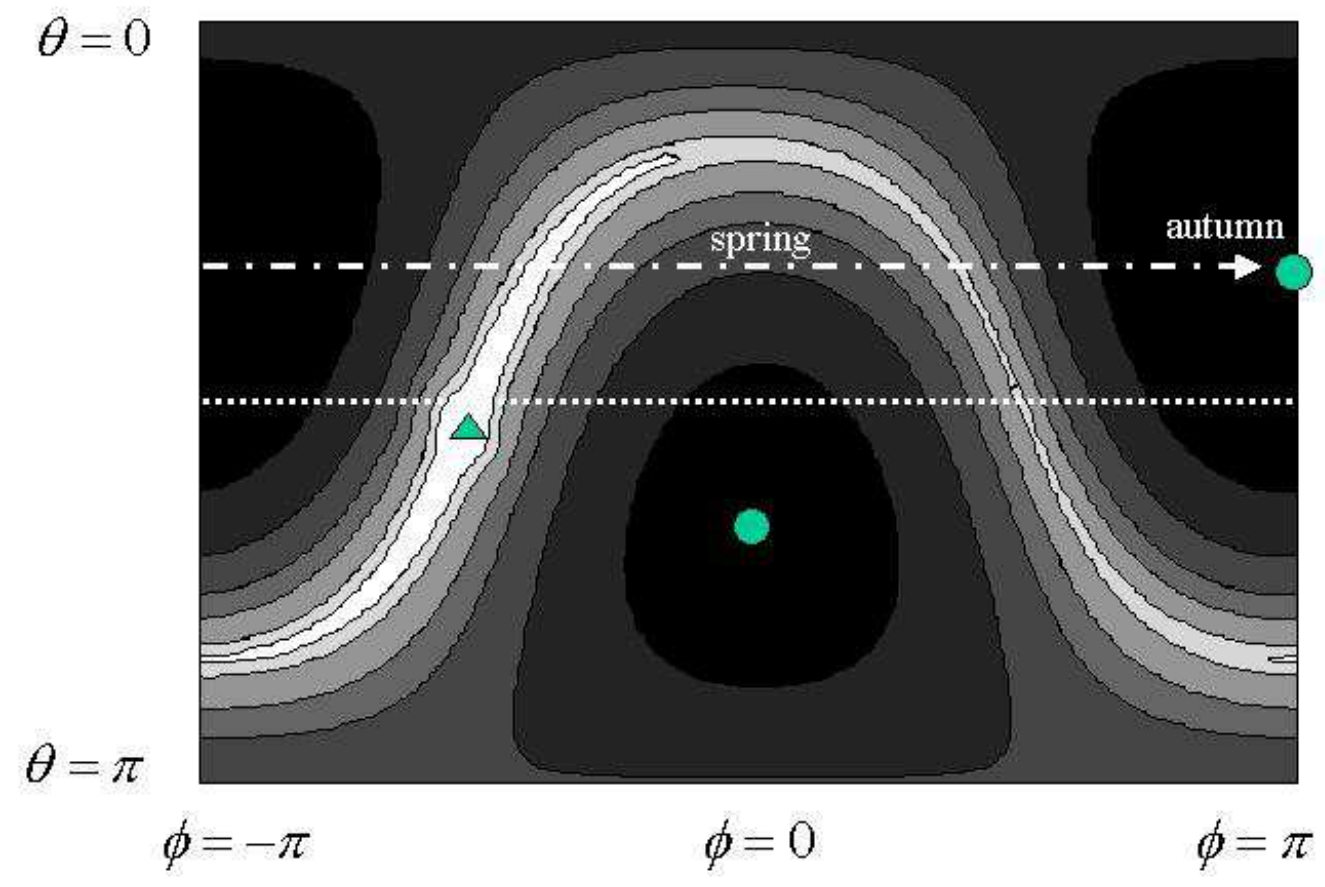

FIG. 3: The gravitational wave luminosity $B(\theta, \phi)$ on the sky with the ecliptic coordinate. The BGS model is used for the Galactic binary distribution. The direction of the Galactic center $\left(\theta_{G C}, \phi_{G C}\right)$ is given by the triangle, and the Galactic poles by circles. The contours correspond to $\log _{10}\left(B(\theta, \phi) / B\left(\theta_{G C}, \phi_{G C}\right)\right)=-2$ (white) to -3 (black) with interval 0.2 . The $Z_{D}$-axis of LISA moves along the dash-dotted line. 


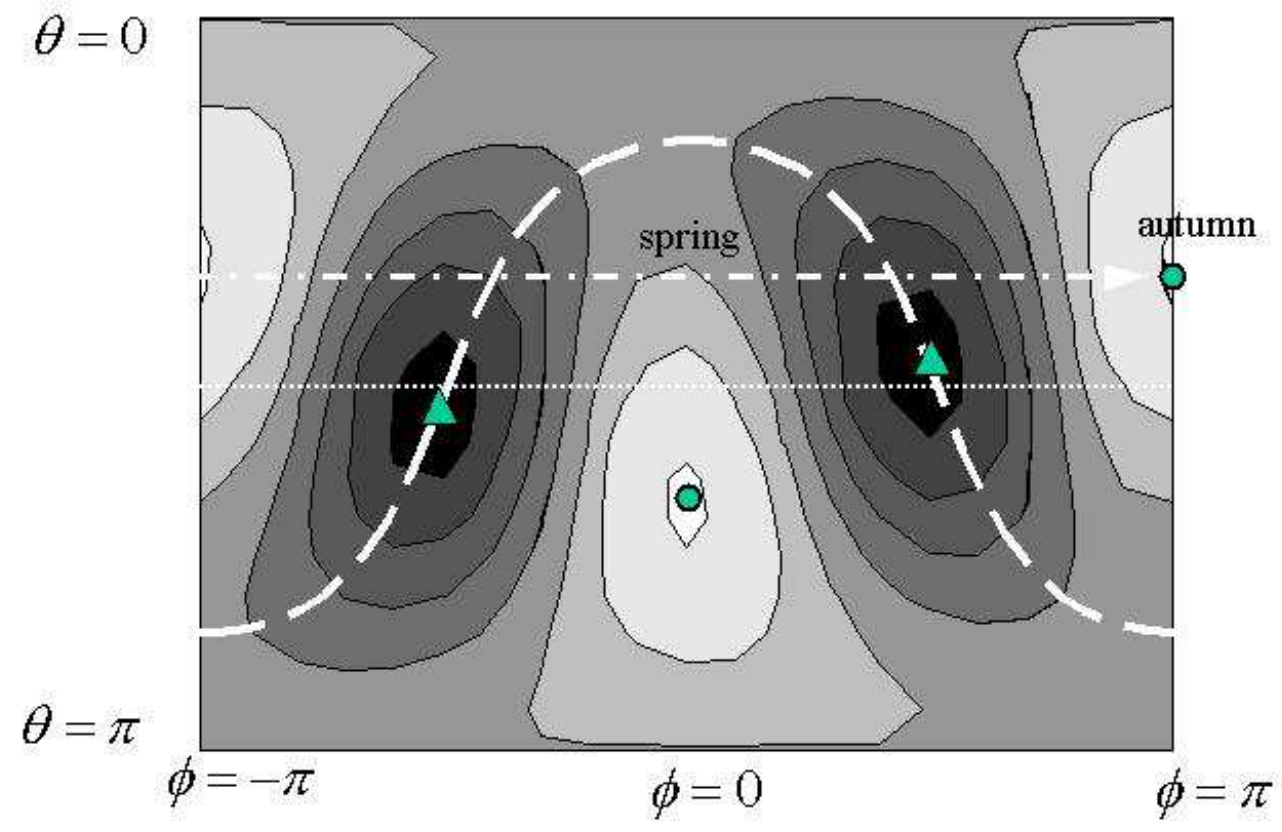

FIG. 4: All sky effective noise map in the ecliptic coordinate. The effective noise $S_{\text {eff }}^{B} / S_{\text {iso }}^{B}$ is plotted as a function of the orientation $(\theta, \phi)$ of the $Z_{D}$-axis of the detector. Contours correspond to $S_{\text {eff }}^{B} / S_{\text {iso }}^{B}=0.4$ (white) to 1.6 (black) with 0.2 interval. The dotted line is the ecliptic plane. The $Z_{D}$-axis of LISA moves along the dash-dotted line. The direction of the Galactic center and Galactic poles are given by the triangles and circles. The Galactic disk plane is given by the long dashed line.

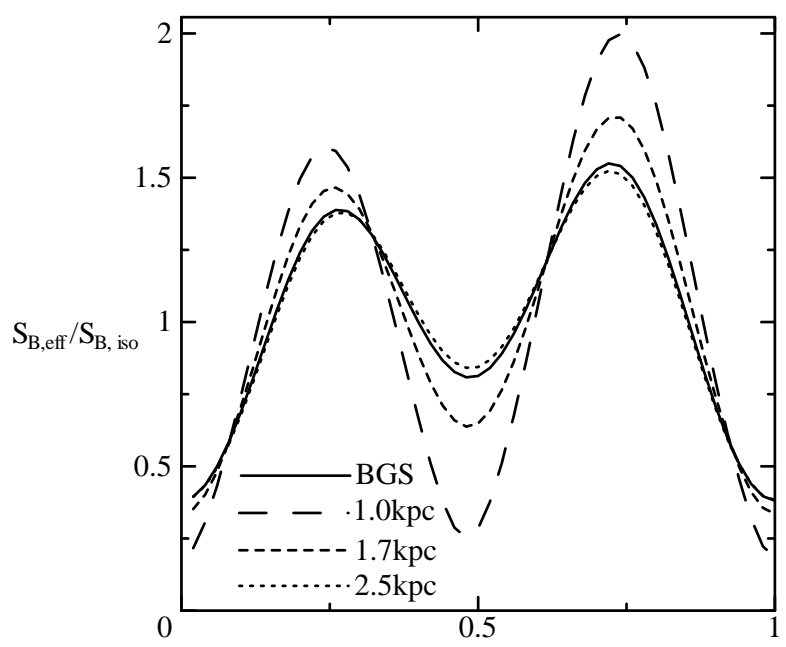

$\mathrm{T}[\mathrm{yr}]$

FIG. 5: Annual modulation of the effective noise $S_{\text {eff }}^{B} / S_{i s o}^{B}$ as a function of the orbital phase $T$ of LISA. The solid line is for the BGS model. Other three curves correspond to the different scale length $R_{s}$ of the Galactic distribution (3.7). 


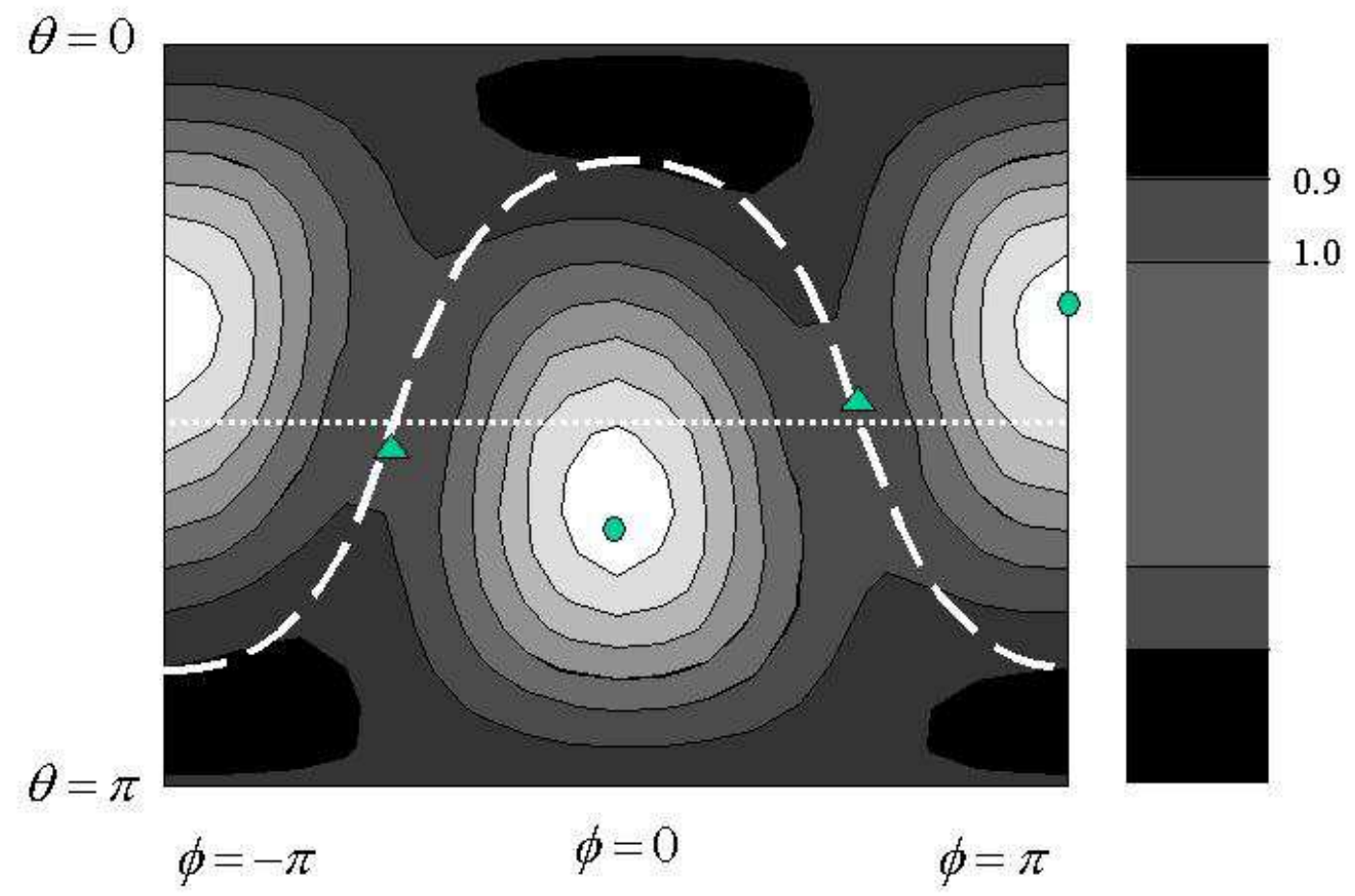

FIG. 6: Directional dependence of $S N R^{2}$ in the ecliptic coordinate after $1 y$ r integration. The left panel is the ratio $G_{3}(\theta, \phi) / G_{1}$. The right small panel is $G_{2}(\theta) / G_{1}$ that does not depend on the azimuthal angle $\phi$ as eq. (4.2). Contours correspond to $S_{\text {eff }}^{B} / S_{\text {iso }}^{B}=0.9$ (black) to 1.5 (white) with 0.1 interval. The directions of the Galactic poles are given by the circles. The Galactic disk plane is given by the long dashed line. By definition this line is $90^{\circ}$ apart from the poles. This map does not depend on the possible two configurations of LISA. 